\title{
Birthweight Extremes and Neonatal and Childhood Outcomes after Preterm Premature Rupture of Membranes
}

\author{
Matthew R. Grace, MD ${ }^{1}$ \\ Tracy A. Manuck, MD ${ }^{1,2}$ \\ ${ }^{1}$ Division of Maternal Fetal Medicine, Department of Obstetrics and \\ Gynecology, University of North Carolina School of Medicine, \\ Chapel Hill, North Carolina \\ 2 Division of Maternal Fetal Medicine, Department of Obstetrics and \\ Gynecology, University of Utah, Salt Lake City, Utah
}

\author{
Michael W. Varner, MD² Kim Boggess, MD ${ }^{1}$
}

Am J Perinatol 2016;33:1138-1144.

\begin{abstract}
Address for correspondence Matthew R. Grace, MD, Division of Maternal Fetal Medicine, Department of Obstetrics and Gynecology, University of North Carolina School of Medicine, 3010 Old Clinic Building, CB\#7516, Chapel Hill, NC 27599-7516

(e-mail: matthew_grace@med.unc.edu).
\end{abstract}

\begin{abstract}
\section{Keywords}

- small for gestational age

- large for gestational age

- preterm premature rupture of membranes

- neonatal morbidity

- childhood morbidity

Objective To determine the association between birthweight extremes and risk of adverse neonatal and childhood outcomes following preterm premature rupture of membranes (PPROM).

Study Design This is a secondary analysis of data from the Beneficial Effects of Antenatal Magnesium Sulfate Trial. Women with nonanomalous singletons and PPROM delivering $\geq 24.0$ weeks were included. Birthweight was classified as small for gestational age (SGA), appropriate for gestational age (AGA), or large for gestational age (LGA). Composite severe neonatal morbidity and childhood outcomes at age 2 , were compared between these groups.

Results One thousand five hundred and ninety-eight infants were included (58 SGA, 1,354 AGA, and 186 LGA). There was an inverse relationship between birthweight and rate of composite major neonatal morbidity (55.2\% of SGA, 31.5\% of AGA, $18.3 \%$ of LGA, $p<0.001)$. Former-SGA children were more likely to be diagnosed with major composite childhood morbidity at age 2 (25.9\% of SGA, $8.3 \%$ of AGA, 5.9\% of LGA, $p<0.001)$. In multivariate models, LGA infants had improved initial neonatal outcomes compared with AGA infants (adjusted odds ratio [aOR], 0.44; 95\% confidence interval [Cl], 0.28-0.71; $p=0.001)$.

Conclusion Among infants delivered following PPROM, those who were LGA at delivery had improved composite adverse neonatal outcomes. SGA increases the risk of severe neonatal morbidity, early childhood death, and moderate/severe cerebral palsy at age 2 .
\end{abstract}

Preterm premature rupture of membranes (PPROM) is the leading identifiable cause of preterm birth and affects $3 \%$ of all pregnancies. ${ }^{1}$ Following PPROM, women are at high risk of additional pregnancy complications including preterm labor, placental abruption, and chorioamnionitis. ${ }^{2,3}$ Women with PPROM between 23 and 34 weeks of gestation and without evidence of other immediate complications are typically

received

May 21, 2016

accepted

May 25, 2016

published online

July 1,2016

administered a course of antenatal corticosteroids, intravenous magnesium sulfate for neuroprotection (up to $32 \mathrm{wk}$ ), and latency antibiotics, and are hospitalized until delivery. ${ }^{4-6}$

Premature infants, including those born after PPROM, are at risk of both short-term and long-term morbidity and death. Long-term complications of prematurity persist into childhood and beyond, and may include neurodevelopmental

Copyright $\odot 2016$ by Thieme Medical Publishers, Inc., 333 Seventh Avenue, New York, NY 10001, USA. Tel: +1(212) 584-4662.
DOI http://dx.doi.org/ 10.1055/s-0036-1584898. ISSN 0735-1631. 
disabilities, failure-to-thrive, vision and hearing impairment, and cerebral palsy $(\mathrm{CP})^{7,8}$ Although gestational age at birth is typically regarded as the most important factor influencing the risk of both short- and long-term morbidity, other risk factors are known to affect outcomes, including birthweight, gender, and medication exposure (e.g., antenatal corticosteroids, magnesium sulfate) prior to delivery. ${ }^{8,9}$ Birthweight estimates are often used along with gestational age to counsel families regarding infant outcome following preterm delivery and in decisions regarding antenatal testing and intervention, mode of delivery, and postnatal intervention. ${ }^{10,11}$ The correlation between ultrasound (US)-based estimates of fetal weight in the setting of PPROM and postnatal birthweight is understudied, and is likely hindered by the high incidence of oligohydramnios and anhydramnios in this population.

The extent to which extremes of birthweight (e.g., being large for gestational age [LGA] or small for gestational age [SGA] compared with birthweight that is appropriate for gestational age [AGA]) affect postnatal outcomes among infants born after PPROM is unknown. We sought to determine the relationship between birthweight extremes (LGA, SGA) and major neonatal and childhood morbidity. We hypothesized that compared with AGA infants delivered after PPROM, LGA infants have lower prevalence of adverse neonatal and childhood outcomes and that SGA infants have higher incidence of adverse neonatal and childhood outcomes.

\section{Materials and Methods}

This is a secondary analysis of a multicenter, randomized clinical trial of antenatal magnesium sulfate versus placebo administered to women at imminent risk for early preterm birth ( $<32 \mathrm{wk}$ ) to prevent death and CP in their offspring. The inclusion criteria and randomization protocol for the original trial have been previously reported. ${ }^{12}$ Briefly, the original study recruited women from 1997 to 2004 at 20 centers, who were at high risk of preterm delivery between 24 to 31 weeks' gestation for any indication. The majority of enrolled women had pregnancies complicated by PPROM. Per the original protocol, study staff evaluated surviving infants at 6,12 , and 24 months corrected gestational age. Written, informed consent was obtained at the time of the original study from each participant. This secondary analysis used a de-identified dataset, and after review by our Institutional Review Board (IRB) at the University of North Carolina, was considered exempt from IRB oversight.

For the purposes of this secondary analysis, we included women with singleton, nonanomalous gestations with a diagnosis of PPROM who delivered at 24 weeks 0 days through 34 weeks and 6 weeks. Gestational age was determined from standard algorithms using last menstrual period and US criteria. ${ }^{13}$ Neonatal birthweight was classified as AGA (10-90 percentile), LGA ( $\geq 90$ percentile), or SGA ( $<10$ percentile) based upon gender- and gestational age-specific contemporary national norms. ${ }^{14}$ The primary neonatal outcome was a composite of severe neonatal morbidity, defined as one or more of the following; neonatal sepsis, grade III or IV intraventricular hemorrhage, periventricular leukomalacia, severe (stage II or III) necrotizing enterocolitis, bronchopulmonary dysplasia (BPD), and/or death during the initial hospitalization. The primary childhood outcome was a composite of stillbirth or infant death by 1 year of age, or moderate or severe $\mathrm{CP}$, as assessed at the final follow-up visit, prespecified to occur at 2 years of age. Secondary outcomes included individual components of each of the two primary composite outcomes and Bayley Scales of Infant Development II Mental Development Index (MDI) and/or Psychomotor Development Index (PDI) greater than 2 standard deviation (SD) below the mean at age 2. We also compared weight at the time of childhood follow-up assessments to determine whether formerly-SGA infants remained smaller than their formerly-AGA and formerly-LGA counterparts in early childhood.

Bivariate analyses were by chi-square or Fisher's exact for categorical variables, analysis of variance or Kruskal-Wallis equality of proportions rank test when there were greater than two categories. The Wilcoxon-type test for trend as described by Cuzick was utilized to assess trend across ordered groups. ${ }^{15}$ Multivariable analysis was performed using stepwise backward elimination logistic regression, and factors with $p<0.20$ remained in final models. All data were analyzed using STATA version 13.1 (College Station, TX). In the final bivariate and multivariable analyses a $p$-value of $<0.05$ was considered significant.

\section{Results}

From the original cohort of 2,241 women, 1,598 women and their infants met inclusion criteria (- Fig. 1). Of these, 58 (3.6\%) were SGA, 1,354 (84.7\%) were AGA, and 186 (11.6\%) were LGA. Demographic characteristics are compared by birthweight group in - Table 1. Groups were different with regard to likelihood of diabetes mellitus diagnosis and prepregnancy body mass index (-Table $\mathbf{1}$ ).

- Table 2 shows the pregnancy characteristics of our study cohort. Overall, 494 women (30.9\%) had a first trimester US ( $<14$ week-gestation), which did not vary by birthweight group ( - Table 2). Other pregnancy dating criteria, were also similar between groups (-Table 2). Women with SGA infants had PPROM, and subsequent delivery at earlier gestational ages than women with AGA and LGA infants; they were also more likely to deliver by cesarean and require a cesarean delivery for fetal intolerance of labor (-Table 2 ).

In bivariate analyses, there was an inverse relationship between neonatal outcomes and birthweight group (-Table 3). SGA infants had a higher prevalence of composite major neonatal morbidity compared with AGA and LGA infants (55.2\% vs $31.5 \%$ vs $18.3 \%$, respectively, $p<0.001$ ). This effect was driven by several components of the composite outcome (death prior to initial hospital discharge, BPD, and neonatal sepsis). Former-SGA children were also more likely to be diagnosed with major composite childhood morbidity at age 2 compared with former-AGA and former-LGA children ( $25.9 \%$ vs $8.3 \%$ vs $5.9 \%$, respectively, $p<0.001$ ), (- Table 3 ). Surviving former-SGA children were also more likely than former-AGA or former-LGA children to have Bayley II MDI scores greater than 2 SD below the mean ( $24.4 \%$ vs $16.9 \%$ vs $10.3 \%$, respectively, $p=0.043$ ). 


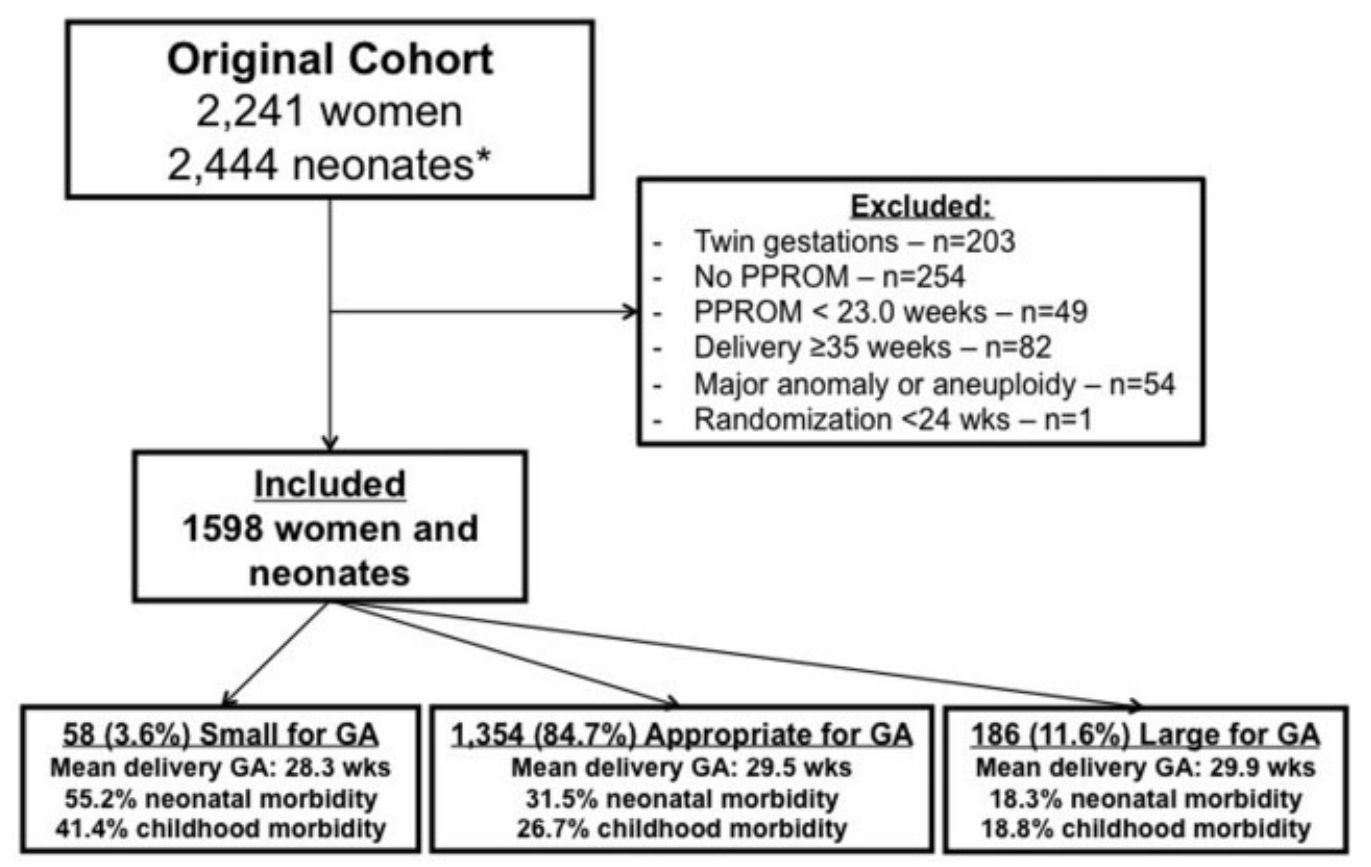

Fig. 1 Study enrollment. GA, gestational age; PPROM, preterm premature rupture of membranes.*There were 203 twin pregnancies.

In multivariable models, several factors remained associated with major neonatal morbidity ( - Table 4). After controlling for delivery gestational age, compared with AGA infants, LGA infants were less likely (aOR, 0.44; 95\% CI, $0.28-0.71 ; p=0.001$ ), and SGA infants were more likely,
(aOR, $2.11 ; 95 \% \mathrm{CI}, 0.98-4.56 ; p=0.057$ ) to have a composite adverse outcome. However, former-SGA infants were more likely to develop major childhood morbidity at age 2 (aOR, 2.63; 95\% CI, 1.27-5.45; $p=0.009$ ), compared with AGA infants, whereas those former-LGA infants had similar risk

Table 1 Demographic characteristics, stratified by birthweight group

\begin{tabular}{|c|c|c|c|c|}
\hline Characteristic & $\begin{array}{l}\text { Small for } \\
\text { gestational age } \\
N=58\end{array}$ & $\begin{array}{l}\text { Appropriate for } \\
\text { gestational age } \\
N=1,354\end{array}$ & $\begin{array}{l}\text { Large for } \\
\text { gestational age } \\
N=186\end{array}$ & $p$-value \\
\hline Maternal age (y; median, IQR) & $25.5(22-31)$ & $26(21-30)$ & $27(22-32)$ & 0.023 \\
\hline $\begin{array}{l}\text { Prepregnancy body mass index } \\
\left(\mathrm{kg} / \mathrm{m}^{2} ; \text { median, IQR) }\right.\end{array}$ & $\begin{array}{l}25.2 \\
(20.6-30.1)\end{array}$ & $\begin{array}{l}24.4 \\
(21.2-29.8)\end{array}$ & $\begin{array}{l}26.7 \\
(22.3-30.9)\end{array}$ & 0.007 \\
\hline \multicolumn{5}{|l|}{ Race/ethnicity ( $n$; \%) } \\
\hline African-American & $36(62.1)$ & $612(45.2)$ & $77(41.4)$ & \multirow[t]{3}{*}{0.302} \\
\hline Caucasian & 15 (25.9) & $499(36.8)$ & $74(39.8)$ & \\
\hline Hispanic & $6(10.3)$ & $211(15.6)$ & $31(16.7)$ & \\
\hline Married ( $n ; \%)$ & $26(44.8)$ & $635(47.0)$ & $101(54.3)$ & 0.160 \\
\hline High school education or higher ( $n$; \%) & $35(61.4)$ & $865(63.9)$ & $130(69.9)$ & 0.248 \\
\hline Alcohol use during pregnancy $(n ; \%)$ & $6(10.3)$ & $130(9.6)$ & $18(9.7)$ & 0.982 \\
\hline Tobacco use during pregnancy $(n ; \%)$ & $15(25.9)$ & $411(30.3)$ & $48(25.8)$ & 0.364 \\
\hline Drug use during pregnancy ( $n$; \%) & $4(6.9)$ & $154(11.4)$ & $18(9.7)$ & 0.469 \\
\hline Nulliparous (n; \%) & $25(43.1)$ & $471(34.8)$ & $62(33.3)$ & 0.381 \\
\hline \multicolumn{5}{|l|}{ Diabetes $(n ; \%)$} \\
\hline Pregestational & $1(1.7)$ & $19(1.4)$ & $4(2.2)$ & 0.550 \\
\hline Gestational & $3(5.2)$ & $39(2.9)$ & $12(6.5)$ & 0.030 \\
\hline Any & $4(6.9)$ & $58(4.3)$ & $16(8.6)$ & 0.027 \\
\hline Previous preterm birth ( $n ; \%)$ & $14(24.1)$ & 391 (28.9) & $48(25.8)$ & 0.530 \\
\hline
\end{tabular}

Abbreviation: IQR, interquartile range. 
Table 2 Current pregnancy and intrapartum characteristics, stratified by birthweight group

\begin{tabular}{|c|c|c|c|c|}
\hline Characteristic & $\begin{array}{l}\text { Small for } \\
\text { gestational age } \\
N=58\end{array}$ & $\begin{array}{l}\text { Appropriate for } \\
\text { gestational age } \\
N=1,354\end{array}$ & $\begin{array}{l}\text { Large for } \\
\text { gestational age } \\
N=186\end{array}$ & $p$-value \\
\hline \multicolumn{5}{|l|}{ Pregnancy dating criteria } \\
\hline Mean gestational age at first ultrasound (wk; \pm SD) & $18.8 \pm 6.8$ & $18.2 \pm 7.2$ & $18.8 \pm 8.6$ & 0.537 \\
\hline First ultrasound was prior to 14 wk gestation ( $n$; \%) & $15(25.9)$ & $414(30.6)$ & $65(35.0)$ & 0.336 \\
\hline Patient had a missing last menstrual period date $(n ; \%)$ & $5(8.6)$ & $122(9.0)$ & $20(10.8)$ & 0.734 \\
\hline Patient reported a "sure" last menstrual period date ( $n ; \%)$ & $36(62.1)$ & $792(58.5)$ & $107(57.5)$ & 0.828 \\
\hline Mean gestational age at PPROM (wk; \pm SD) & $26.9 \pm 3.1$ & $28.0 \pm 2.5$ & $28.4 \pm 2.4$ & 0.005 \\
\hline Randomized to receive magnesium sulfate $(n ; \%)$ & $29(50)$ & $670(49.5)$ & $80(43.0)$ & 0.253 \\
\hline Received antenatal antibiotics ( $n$; \%) & $58(100)$ & $1,308(96.6)$ & $180(96.8)$ & 0.361 \\
\hline Received antenatal corticosteroids ( $n$; \%) & $57(98.3)$ & $1,322(97.6)$ & $181(97.3)$ & 0.912 \\
\hline Latency between PPROM and delivery (d; median, IQR) & $6.4(3.8-13.1)$ & $6.9(3.3-14.1)$ & $6.6(2.6-15.1)$ & 0.915 \\
\hline Chorioamnionitis ( $n$; \%) & $8(13.8)$ & $174(12.9)$ & $26(14.0)$ & 0.898 \\
\hline Preeclampsia $(n ; \%)$ & $1(1.7)$ & $8(0.6)$ & $1(0.5)$ & 0.386 \\
\hline Meconium stained amniotic fluid $(n ; \%)$ & $3(5.2)$ & $31(2.3)$ & $3(1.6)$ & 0.261 \\
\hline Cesarean delivery $(n ; \%)$ & $31(53.5)$ & $510(37.7)$ & $56(30.1)$ & 0.005 \\
\hline Cesarean delivery for fetal intolerance of labor ( $n$; \%) & $14 / 31(45.2)$ & $218 / 510(42.8)$ & $14 / 56(25)$ & 0.034 \\
\hline Cesarean delivery for malpresentation ( $n$; \%) & $17 / 31(54.8)$ & $251 / 510(49.2)$ & $31 / 56(55.4)$ & 0.589 \\
\hline Mean gestational age at delivery (wk; \pm SD) & $28.3 \pm 3.1$ & $29.5 \pm 2.6$ & $29.9 \pm 2.4$ & 0.004 \\
\hline
\end{tabular}

Abbreviations: IQR, interquartile range; PPROM, preterm premature rupture of membranes; SD, standard deviation.

compared with former-AGA children (aOR, 0.74 ; $95 \% \mathrm{CI}, 0.35$ $1.55 ; p=0.425$ ), - Table 5. Male gender was associated with worse outcomes both during the initial neonatal period and at age 2 ( - Tables 4 and 5 ).

Finally, we compared the median birthweight in kilograms $(\mathrm{kg})$ and at each childhood follow-up visit, stratified by birthweight group. Children's weights were recorded at the three prespecified follow-up visits and the timing of the follow-up visits did not vary by birthweight group (median of 6,13 and $25 \mathrm{mo}$ ), corrected for prematurity. At each followup visit, the former-SGA children remained significantly lighter than their former-AGA counterparts, who in turn were significantly lighter than the former-LGA children $(p<0.001$ for all time points studied, - Fig. 2 ).

\section{Comment}

In this large, prospectively collected cohort of women with pregnancies complicated by PPROM, we found that infant birthweight classification is associated with both adverse initial neonatal outcomes and adverse childhood neurodevelopmental outcomes at age 2 . These differences appeared most significant for those SGA at delivery, as adverse outcomes persisted to early childhood. The apparent advantage conferred shortly after birth to LGA infants appeared to be short-lived, as neurodevelopmental outcomes were similar between former-LGA and former-AGA children when they were evaluated at age 2 .
These findings are consistent with limited previous reports examining the effect of birthweight on preterm morbidity and mortality, particularly among neonates with an antenatal diagnosis of intrauterine fetal growth restriction. Multiple prior studies have demonstrated adverse effects of intrauterine fetal growth restriction (diagnosed antenatally) on initial childhood outcomes as well as neurodevelopmental impairment. ${ }^{16-18}$ However, the majority of prior studies have focused specifically on antenatal growth restriction and US parameters, and have used this information for decision making. ${ }^{19,20}$ This cohort, where PPROM was the obstetric diagnosis for all women studied, likely represents a different population, given different underlying pathophysiology contributing to premature membrane rupture, and provides valuable information regarding anticipated postnatal outcomes among babies delivered following this complication.

Furthermore, we report information on infants that were LGA at delivery. Although these infants experienced lower morbidity in the neonatal period, this benefit was transient, as they experienced similar neurodevelopmental outcomes at age 2 to former-AGA children. We hypothesize that LGA infants might have improved outcomes due to reduced need for transfusion, and also reduced temperature instability, known risk factors for neonatal morbidity. ${ }^{21,22}$ Unfortunately, our study design does not permit us to answer these mechanistic questions directly. For all groups, size at birth was highly correlated with weight across multiple follow-up visits through 2 years of age. The longer term (e.g., adult) 
Table 3 Neonatal outcomes, stratified by birthweight group

\begin{tabular}{|c|c|c|c|c|}
\hline Characteristic & $\begin{array}{l}\text { Small for } \\
\text { gestational age } \\
N=58\end{array}$ & $\begin{array}{l}\text { Appropriate for } \\
\text { gestational age } \\
N=1,354\end{array}$ & $\begin{array}{l}\text { Large for } \\
\text { gestational age } \\
N=186\end{array}$ & $p$-value \\
\hline Male fetus (n; \%) & $35(60.3)$ & $726(53.6)$ & $90(48.4)$ & 0.222 \\
\hline Median birthweight (kg; IQR) & $0.73(0.57-1.13)$ & $1.35(0.99-1.67)$ & $1.79(1.35-2.10)$ & $<0.001$ \\
\hline 5-min Apgar score < 7 ( $n$; \%) & $17(29.3)$ & $252(18.6)$ & $25(13.5)$ & 0.023 \\
\hline $\begin{array}{l}\text { Birth head circumference }<10 \% \text { for } \\
\text { gestational age }(n ; \%)\end{array}$ & $31(56.4)$ & $96(7.2)$ & $1(0.6)$ & $<0.001$ \\
\hline Birth length $<10 \%$ for gestational age $(n ; \%)$ & $27(47.4)$ & $65(4.8)$ & $1(0.6)$ & $<0.001$ \\
\hline Composite major neonatal morbidity ( $n$; \%) & $32(55.2)$ & $426(31.5)$ & $34(18.3)$ & $<0.001$ \\
\hline Death prior to initial hospital discharge $(n ; \%)$ & $10(17.9)$ & $63(4.7)$ & $4(2.2)$ & $<0.001$ \\
\hline Necrotizing enterocolitis, stage II or III (n; \%) & $0(0)$ & $66(4.9)$ & $4(2.2)$ & 0.055 \\
\hline Bronchopulmonary dysplasia ( $n$; \%) & $17(30.4)$ & $233(17.3)$ & $15(8.2)$ & $<0.001$ \\
\hline Confirmed neonatal sepsis ( $n$; \%) & $14(25.0)$ & $222(16.5)$ & $15(8.2)$ & 0.002 \\
\hline Intraventricular hemorrhage, grade III or IV ( $n$; \%) & $1(1.9)$ & $21(1.6)$ & $3(1.7)$ & 0.981 \\
\hline Periventricular leukomalacia ( $n$; \%) & $1(1.9)$ & $21(1.6)$ & $3(1.7)$ & 0.981 \\
\hline Composite major childhood morbidity ( $n$; \%) & $15(25.9)$ & $112(8.3)$ & $11(5.9)$ & $<0.001$ \\
\hline Stillbirth or infant death $(n ; \%)$ & $14(24.6)$ & $92(7.1)$ & $8(4.4)$ & $<0.001$ \\
\hline Moderate or severe cerebral palsy at age $2^{a}$ & $1 / 43(1.8)$ & $21 / 1,201(1.6)$ & $3 / 172(1.7)$ & 0.790 \\
\hline Bayley MDI score $>2$ SD below the mean ${ }^{a}$ & $10 / 41(24.4)$ & $185 / 1,096(16.9)$ & $16 / 155(10.3)$ & 0.043 \\
\hline Bayley PDI score $>2$ SD below the mean ${ }^{a}$ & $4 / 41(9.8)$ & $166 / 1,102(15.1)$ & $19 / 157(12.1)$ & 0.417 \\
\hline
\end{tabular}

Abbreviations: IQR, interquartile range; MDI, mental development index; PDI, psychomotor development index; SD, standard deviation.

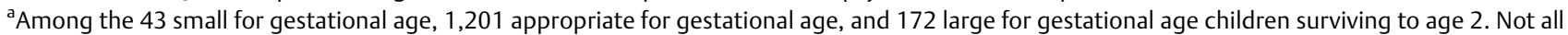
assessments were completed for each child; numerator for each parameter reflects number of living children with available data.

implications of LGA and SGA birthweight for gestational age growth patterns is unknown.

In this population, traditional risk factors for aberrant fetal growth were uncommon; for example, use of tobacco and illicit drugs was low and did not vary by birthweight group. Pre-eclampsia was also uncommon. Previous studies examining the rate of fetal growth following PPROM or after the onset of spontaneous preterm labor have noted reduced growth velocity, suggesting that slowing of fetal growth may participate in the mechanism of preterm labor. ${ }^{23,24}$ Significant placental lesions (e.g., syncytiotrophoblast knot- ting, villous fibrosis, avascular terminal villi, accelerated villous maturation) have been associated with abnormal fetal growth in the setting of preterm delivery, supporting the theory that the development of these complications may be a chronic process. ${ }^{25,26}$

Our finding of a greater proportion (11.6\%) of LGA compared with SGA infants (3.6\%) is of uncertain significance, and warrants further investigation in other cohorts. The rate of SGA is uncommonly reported following PPROM, but the incidence in our population was similar to that reported in a large cohort $(n=4,102)$ of women delivering $<32$ weeks,

Table 4 Multivariate logistic regression model for major neonatal morbidity

\begin{tabular}{|l|l|l|l|}
\hline Characteristic & OR & $95 \% \mathrm{Cl}$ & $p$-value \\
\hline Delivery gestational age (per 1 wk interval) & 0.50 & $0.47-0.54$ & $<0.001$ \\
\hline Male infant & 1.52 & $1.15-2.00$ & 0.003 \\
\hline Maternal education (per y completed) & 1.05 & $0.99-1.11$ & 0.073 \\
\hline Infant size at delivery & & & \\
\hline Small for gestational age & 2.11 & $0.98-4.56$ & 0.057 \\
\hline Appropriate for gestational age & 1.00 & Ref & Ref \\
\hline Large for gestational age & 0.44 & $0.28-0.71$ & 0.001 \\
\hline
\end{tabular}

Abbreviations: $\mathrm{Cl}$, confidence interval; OR, odds ratio; Ref, reference group.

Note: Other factors considered in initial model but removed from final models due to $p>0.20$ included maternal body mass index, randomization to magnesium sulfate, maternal smoking, and maternal diabetes mellitus. 
Table 5 Multivariate logistic regression model for major childhood morbidity at age 2

\begin{tabular}{|l|l|l|l|}
\hline Characteristic & OR & $95 \% \mathrm{Cl}$ & -value \\
\hline Male child & 1.63 & $1.08-2.47$ & 0.021 \\
\hline $\begin{array}{l}\text { Delivery gestational age } \\
\text { (per 1 wk interval) }\end{array}$ & 0.63 & $0.57-0.69$ & $<0.001$ \\
\hline $\begin{array}{l}\text { Prepregnancy maternal body } \\
\text { mass index } \\
\text { (per 1 unit increase) }\end{array}$ & 1.02 & $0.99-1.05$ & 0.150 \\
\hline Infant size at delivery & 2.63 & $1.27-5.45$ & 0.009 \\
\hline Small for gestational age & 1.00 & Ref & Ref \\
\hline $\begin{array}{l}\text { Appropriate for } \\
\text { gestational age }\end{array}$ & 0.74 & $0.35-1.55$ & 0.425 \\
\hline Large for gestational age
\end{tabular}

Abbreviations: $\mathrm{Cl}$, confidence interval; OR, odds ratio; Ref, reference group. Note: Other factors considered in initial model but removed from final models due to $p>0.20$ included randomization to magnesium sulfate, maternal education level, maternal smoking, and maternal diabetes mellitus.

where $4.7 \%$ delivered after PPROM were SGA. ${ }^{27}$ A phenotype of rapid fetal growth prior to the occurrence of late preterm birth has been described; we speculate that perhaps this may have contributed to the lower rate of SGA and the higher rate of LGA even though this cohort was primarily earlier preterm. ${ }^{28}$ Further mechanistic investigations are needed, and placental pathology information will be crucial to unraveling underlying etiologies of growth disturbances in these fetuses.

Our study has several strengths. This is a large, well-defined cohort of women with PPROM, diagnosed in a standardized fashion. Given that the original study was not focused on fetal growth per se, women were not excluded due to suspected normal or abnormal fetal growth, and we had the distinct advantage of being able to evaluate the impact of SGA and LGA

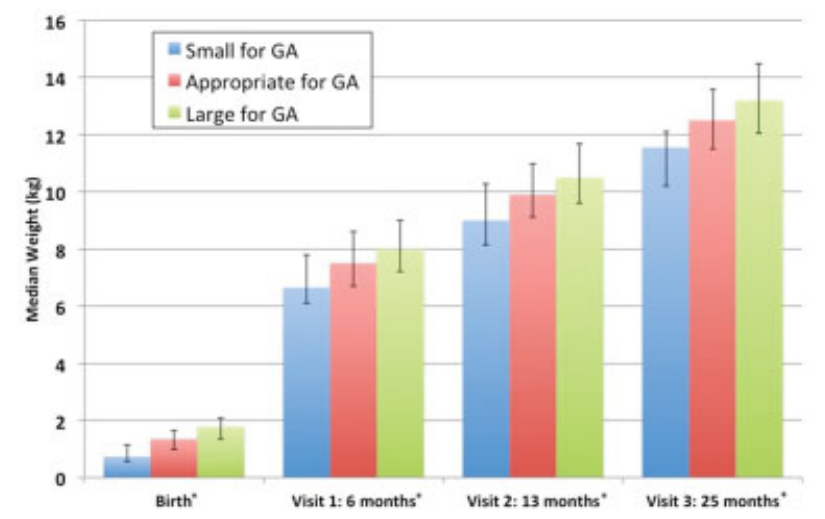

Fig. 2 Median weight at birth and at subsequent follow-up visits, stratified by birthweight group. Error bars represent interquartile range. Ages listed for follow-up visits were at corrected gestational ages. Timing of visits did not differ by birthweight group. Follow-up weight examination at visit 1 included 44 formerly-SGA, 1,219 formerly- AGA, and 174 formerly- LGA, at visit 2 included 40 formerly-SGA, 1,130 formerly-AGA, and 167 formerly-LGA, and at visit 3 included 34 formerly-SGA, 847 formerly-AGA, and 120 formerly-LGA children. AGA, appropriate for gestational age; LGA, large for gestational age; SGA, small for gestational age. ${ }^{*} p<0.001$ for each visit. from within the same cohort. Neonatal and childhood outcomes were also standardized and were rigorously prospectively assessed by trained research staff. We had detailed pregnancy dating information available, reducing the likelihood that infants were misclassified as SGA or LGA due to incorrect pregnancy dating. Furthermore, incorporation of early childhood outcomes at age 2 allowed for more comprehensive assessment regarding the impact of size on outcomes.

Our analysis is not without limitation. As with all secondary analyses, we were limited to the data previously collected as part of the main trial. We did not have information regarding placental pathology, and had minimal details regarding US assessments following PPROM. We also had very limited data regarding estimated fetal weight or amniotic fluid index (AFI) by US following PPROM, and therefore, are unable to examine outcomes based on individual biometric parameters (e.g., abdominal circumference or AFI) or evaluate how various Doppler indices may impact outcomes. The study design did not permit us to examine the mechanism by which SGA contributes to adverse outcomes. Additionally, we were limited by the relatively small number of infants with SGA.

In conclusion, among infants delivered following PPROM, neonatal outcomes were inversely related to size at delivery, with fewer LGA infants experiencing early morbidity compared with AGA and SGA infants. At age 2, former-SGA children had continued adverse effects, with significantly worse neurodevelopmental outcomes compared with former-AGA and formerLGA children. However, the apparent benefit of LGA that was seen during the neonatal period was no longer appreciated in early childhood. These data have implications for counseling patients; future studies should confirm these results and investigate potential mechanisms behind these findings.

Note

This study was presented, in part, in poster format at the 34th annual meeting of the Society for Maternal-Fetal Medicine, New Orleans, LA, February 3 to 8, 2014.

\section{Funding}

This study was funded by the NICHD 5K23HD067224 (Dr. Manuck).

\section{Conflict of Interest}

None.

\section{Acknowledgments}

The authors thank the Eunice Kennedy Shriver National Institute of Child Health and Human Development, the Maternal-Fetal Medicine Units Network, and the Protocol Subcommittee for making this database publically available. The contents of this report represent the views of the authors and do not represent the views of the Eunice Kennedy Shriver National Institute of Child Health and Human Development Maternal-Fetal Medicine Units Network or the National Institutes of Health. 


\section{References}

1 Mercer BM. Preterm premature rupture of the membranes: current approaches to evaluation and management. Obstet Gynecol Clin North Am 2005;32(3):411-428

2 Ananth CV, Oyelese Y, Srinivas N, Yeo L, Vintzileos AM. Preterm premature rupture of membranes, intrauterine infection, and oligohydramnios: risk factors for placental abruption. Obstet Gynecol 2004;104(1):71-77

3 Garite TJ, Freeman RK. Chorioamnionitis in the preterm gestation. Obstet Gynecol 1982;59(5):539-545

4 Mercer BM, Miodovnik M, Thurnau GR, et al; National Institute of Child Health and Human Development Maternal-Fetal Medicine Units Network. Antibiotic therapy for reduction of infant morbidity after preterm premature rupture of the membranes. A randomized controlled trial. JAMA 1997;278(12):989-995

5 Practice bulletins No. 139: premature rupture of membranes. Obstet Gynecol 2013;122(4):918-930

6 Roberts D, Dalziel S. Antenatal corticosteroids for accelerating fetal lung maturation for women at risk of preterm birth. Cochrane Database Syst Rev 2006;(3):CD004454

7 Stoll BJ, Hansen NI, Bell EF, et al; Eunice Kennedy Shriver National Institute of Child Health and Human Development Neonatal Research Network. Neonatal outcomes of extremely preterm infants from the NICHD Neonatal Research Network. Pediatrics 2010;126(3):443-456

8 Eichenwald EC, Stark AR. Management and outcomes of very low birth weight. N Engl J Med 2008;358(16):1700-1711

9 Tyson JE, Parikh NA, Langer J, Green C, Higgins RD; National Institute of Child Health and Human Development Neonatal Research Network. Intensive care for extreme prematurity-moving beyond gestational age. $\mathrm{N}$ Engl J Med 2008;358(16): 1672-1681

10 Raju TN, Mercer BM, Burchfield DJ, Joseph GF Jr. Periviable birth: executive summary of a joint workshop by the Eunice Kennedy Shriver National Institute of Child Health and Human Development, Society for Maternal-Fetal Medicine, American Academy of Pediatrics, and American College of Obstetricians and Gynecologists. Am J Obstet Gynecol 2014;210(5):406-417

11 Rysavy MA, Li L, Bell EF, et al; Eunice Kennedy Shriver National Institute of Child Health and Human Development Neonatal Research Network. Between-hospital variation in treatment and outcomes in extremely preterm infants. N Engl J Med 2015; 372(19):1801-1811

12 Rouse DJ, Hirtz DG, Thom E, et al; Eunice Kennedy Shriver NICHD Maternal-Fetal Medicine Units Network. A randomized, controlled trial of magnesium sulfate for the prevention of cerebral palsy. N Engl J Med 2008;359(9):895-905

13 Carey JC, Klebanoff MA, Hauth JC, et al; National Institute of Child Health and Human Development Network of Maternal-Fetal Medicine Units. Metronidazole to prevent preterm delivery in pregnant women with asymptomatic bacterial vaginosis. $\mathrm{N}$ Engl J Med 2000;342(8):534-540
14 Olsen IE, Groveman SA, Lawson ML, Clark RH, Zemel BS. New intrauterine growth curves based on United States data. Pediatrics 2010;125(2):e214-e224

15 Cuzick J. AWilcoxon-type test for trend. Stat Med 1985;4(1):87-90

16 Murray E, Fernandes M, Fazel M, Kennedy SH, Villar J, Stein A. Differential effect of intrauterine growth restriction on childhood neurodevelopment: a systematic review. BJOG 2015;122(8): 1062-1072

17 Levine TA, Grunau RE, McAuliffe FM, Pinnamaneni R, Foran A, Alderdice FA. Early childhood neurodevelopment after intrauterine growth restriction: a systematic review. Pediatrics 2015; 135(1):126-141

18 von Beckerath AK, Kollmann M, Rotky-Fast C, Karpf E, Lang U, Klaritsch P. Perinatal complications and long-term neurodevelopmental outcome of infants with intrauterine growth restriction. Am J Obstet Gynecol 2013;208(2):130.e1-130.e6

19 García B, Llurba E, Valle L, et al. Do knowledge of uterine artery resistance in the second trimester and targeted surveillance improve maternal and perinatal outcome? UTOPIA study: a randomized controlled trial. Ultrasound Obstet Gynecol 2016;47(6):680-689

20 Gomez-Roig MD, Mazarico E, Sabria J, Parra J, Oton L, Vela A. Use of placental growth factor and uterine artery doppler pulsatility index in pregnancies involving intrauterine fetal growth restriction or preeclampsia to predict perinatal outcomes. Gynecol Obstet Invest 2015;80(2):99-105

21 Buetow KC, Klein SW. Effect of Maintenance of "Normal" Skin Temperature on Survival of Infants of Low Birth Weight. Pediatrics 1964;34:163-170

22 Day RL, Caliguiri L, Kamenski C, Ehrlich F. Body Temperature and Survival of Premature Infants. Pediatrics 1964;34:171-181

23 Bukowski R, Gahn D, Denning J, Saade G. Impairment of growth in fetuses destined to deliver preterm. Am J Obstet Gynecol 2001; 185(2):463-467

24 Hediger ML, Scholl TO, Schall JI, Miller LW, Fischer RL. Fetal growth and the etiology of preterm delivery. Obstet Gynecol 1995;85(2): 175-182

25 Morgan TK, Tolosa JE, Mele L, et al; Eunice Kennedy Shriver National Institute of Child Health and Human Development Maternal-Fetal Medicine Units Network. Placental villous hypermaturation is associated with idiopathic preterm birth. J Matern Fetal Neonatal Med 2013;26(7):647-653

26 Salafia CM, Minior VK, Pezzullo JC, Popek EJ, Rosenkrantz TS, Vintzileos AM. Intrauterine growth restriction in infants of less than thirty-two weeks' gestation: associated placental pathologic features. Am J Obstet Gynecol 1995;173(4):1049-1057

27 Hanke K, Hartz A, Manz M, et al; German Neonatal Network (GNN). Preterm prelabor rupture of membranes and outcome of verylow-birth-weight infants in the German Neonatal Network. PLoS ONE 2015;10(4):e0122564

28 Lampl M, Kusanovic JP, Erez O, et al. Early rapid growth, early birth: accelerated fetal growth and spontaneous late preterm birth. Am J Hum Biol 2009;21(2):141-150 